\title{
Supersymmetry and unification: a status update
}

\author{
Utpal Chattopadhyay and Sourov Roy ${ }^{\mathrm{b}}$ \\ School of Physical Sciences, Indian Association for the Cultivation of Science, 2A \& 2B \\ Raja S.C. Mullick Road, Jadavpur, Kolkata 700 032, India
}

Received 6 October 2020 / Accepted 6 October 2020

Published online 14 December 2020

\begin{abstract}
In this special issue we try to shed light on the current status of supersymmetry in particle physics through a few review articles.
\end{abstract}

The Standard Model (SM) of particle physics has been remarkably successful in describing the fundamental interactions between the elementary particles at presently accessible energies. The predictions of the SM have been verified with increasingly precise tests in high energy particle accelerators like LEP, Tevatron, etc in the past and now at the Large Hadron Collider (LHC). The last missing ingredient of the model, the Higgs boson has also been observed. Thus, the SM is conclusively established as the correct effective theory at energies explored to date. However, from a theoretical point of view, there is a clear notion that the SM cannot be a reasonably complete description of fundamental interactions even if we do not expect to have any connection with gravity. The primary concern is the gauge hierarchy problem. An unnatural amount of cancellation among the one-loop corrections and renormalization counter terms is required to achieve the presently observed Higgs mass value of $125 \mathrm{GeV}$. At the same time, SM cannot address the issue of the existence of dark matter, neutrino mass as well as mixing, matter-antimatter asymmetry. It is true that LHC is yet to observe a definite signal of a candidate of Beyond the Standard Model (BSM) physics. However, the demand for a BSM physics for theoretical consistency as well as the need for addressing various facts of nature like baryon-asymmetry of the universe, neutrino mass and mixing, and the existence of dark matter is too strong to ignore. There are many other areas that could possibly make a BSM physics attractive. This at least includes gauge coupling unification, radiative electroweak symmetry breaking, and Yukawa coupling unification. Additionally, in an ambitious point of view, incorporating grand unified theory (GUT), finding motivated scenarios from extended space-time symmetry, or connecting to string theory in general, all these are still considered to be good features to exist in a BSM physics. SuperSymmetry (SUSY) provided hope with all its inclusiveness and the community, in general, was anxious to see it in the Large Hadron Collider (LHC) at CERN perhaps in its initial period of running. Unfortunately, this did not happen and the LHC at least pushed its existence to higher energy regimes.

In spite of finding no direct evidence of SUSY so far, it is too early to conclude on SUSY since it still remains as the best possible candidate for a BSM physics. We

a e-mail: tpuc@iacs.res.in

b e-mail: tpsr@iacs.res.in 
must remember that SUSY along with its ingredients to bring theoretical consistencies is able to explain the origin of neutrino mass, baryon asymmetry, and the highly possible existence of particle dark matter. The fact that the Higgs boson is discovered with a mass of $125 \mathrm{GeV}$ is interesting since this is within the predicted upper bound of $135 \mathrm{GeV}$ of the Minimal Supersymmetric Standard Model (MSSM). A mass of $125 \mathrm{GeV}$ for Higgs boson is however on the heavy side and is associated with a higher level of fine-tuning for SUSY. This however does not make SUSY unacceptable since it still grossly alleviates the gauge hierarchy problem. We must however remember that accepting SUSY for BSM physics invites a large number of parameters and a search for a suitable mechanism to break the symmetry is essential to give better predictability in this regard. In addition to SUSY breaking mechanisms like the supergravity mediation, gauge mediation, mediation via Super-Weyl anomalies, i.e. the so-called anomaly mediation, mirage mediation there are various string motivated scenarios that were actively pursued over the last decades. The same is true for exploring grand unification or considering extended gauge symmetries in SUSY frameworks. In the middle of the exploration of the concepts like $100 \mathrm{TeV}$ colliders, it is interesting to look back and get a status report of SUSY in relation to the current data.

It is hard to shed light on all the important aspects of supersymmetry in a limited scope. This special issue comprises of a few review articles that are briefly outlined below. It starts with an overview of supersymmetry, supergravity unification, and phenomenological issues of SUSY models in reference [1]. Here, among many details, the author reminded the simple naturalness criterion via hyperbolic branch, covered extended supergravity models, grand unified theories (GUT), etc. Along with the discussions on constraints from muon $g-2$ and dark matter, discovery prospects at high luminosity (HL)-LHC and High Energy (HE)-LHC are also presented. A status review of Natural SUSY models is given in reference [2]. Here the author emphasized the importance of electroweak fine-tuning quantified by a measure $\Delta_{\mathrm{EW}}$ to explore the prospect of SUSY discovery. As mentioned here, all such natural models with low $\Delta_{\mathrm{EW}}$ are typically associated with light higgsinos. The author outlined strategies for searching at the LHC along with its luminosity and energy upgrades, as well as at future electron-positron colliders with $\sqrt{s} \gtrsim 600 \mathrm{GeV}$. Naturalness was further explored in reference [3], but in contrast to reference [2] the authors explored "stringy naturalness". The article involves general considerations from the string theory landscape that favor large values of soft terms, subject to the vacuum selection criteria that electroweak symmetry is properly broken (no charge and/or color breaking (CCB) minima) and the resulting magnitude of the weak scale is not too far from our measured value. The authors explored the collider prospects of natural SUSY at HL and HE-LHC as well as for the International Linear Collider (ILC). Additionally, the authors analyzed SUSY indirect signatures via $(g-2)_{\mu}$ and lepton flavor violation (LFV) while also discussing baryogenesis. The dark matter was considered by focussing on the higgsino and mixed axion plus higgsino-like lightest supersymmetric particle (LSP) scenarios. Reference [4] on the other hand, considered the point of view that dark matter could be the main motivation for studying SUSY models. The author argued that if SUSY exhibits some independent, empirically justifiable motivation the question of naturalness may perhaps be set aside, at least for the time being. In addition to considering massive neutralino (extending to multi-TeV zone) for its indirect DM detection prospects, the author discussed other DM candidates in different SUSY models. This includes right sneutrino $\tilde{\nu}_{R}$ as a DM candidate. The author also extended the discussion briefly to DM candidates in R-parity violating scenarios. Exploring light dark matter $\left(<\frac{m_{h}}{2}\right)$ while staying broadly within MSSM was essentially the chosen theme in the review of reference [5]. Severe constraints come from relic density, direct and indirect DM detection experiments, and collider data. 
Results from Higgs and SUSY searches at the colliders have important implications for the DM mass and couplings. Phenomenological data can however be accommodated rather easily in pMSSM with modified cosmology or in models like NMSSM. The authors considered light neutralino as well as light sneutrino, including the more motivated right sneutrino $\tilde{\nu}_{R}$ as a DM candidate. Search prospects in HL and HELHC were also discussed. Left-Right SUSY models are reviewed in reference [6]. The well-motivated model conserves R-parity. In addition to explaining neutrino mass, the model has the lightest neutralino as well as right sneutrino as DM candidates. The review has an in-depth description of the model as well as phenomenological analyses for dark matter and collider signals at the LHC along with its luminosity and energy upgrades. Reference [7] explores $U(1)^{\prime}$ SUSY models that is helpful toward resolving the $\mu$-problem of MSSM. This is associated with stringency of $Z^{\prime}$-boson mass from LHC data. The author investigates how lowering the mass is possible either in leptophobic models, by employing kinetic mixing, or in models where one considers non-universal $U(1)^{\prime}$ charges. Consistency with dark matter data is analyzed in addition to search prospects for signals in colliders. A brief discussion on axino is made in reference [8]. Here, the author considered axino in the context of dark matter, collider, and as a candidate for sterile neutrino. Reference [9] pursued an alternative route to solve the strong $\mathrm{CP}$ problem by using parity symmetry that bypasses the requirement of axion. The author showed how supersymmetry plays an essential role to keep $\theta$ suppressed by considering the minimal supersymmetric left-right model and its embedding in SUSY SO(10) GUT for fermion masses. Varieties of issues regarding grand unification with or without SUSY are explored in reference [10]. These include dark matter with its stability, baryon asymmetry of the universe, the origin of neutrino and charged fermion masses, vacuum stability of the Higgs potential, and proton decay prediction accessible to ongoing experiments. The last review article [11] of the issue is about the $\mu \nu \mathrm{SSM}$ model. The model addresses several issues at one go. It incorporates right handed neutrinos in the spectra and simultaneously explores neutrino mass, dark matter, baryogenesis, and the $\mu$-problem. The authors explained the above phenomenological issues along with relevant collider signatures.

At the end of this journey, we like to thank B. Ananthanarayan (CHEP, IISc, India) who proposed to us the need to publish a status report on Supersymmetry and Unification. We thank the authors who spent their valuable time contributing to the review articles. Our sincere thanks go to the referees for their careful reading and comments. We would further like to thank Sabine Lehr (Springer) and Sandrine Karpe (EDP Sciences) for all their help regarding our endeavor.

\section{References}

1. P. Nath, Supersymmetry Unification, Naturalness, and Discovery Prospects at HL-LHC and HE-LHC, Eur. Phys. J. Special Topics 229, 3047 (2020)

2. X. Tata, Natural Supersymmetry: Status and Prospects, Eur. Phys. J. Special Topics 229, $3061(2020)$

3. H. Baer, D. Sengupta, S. Salam, K. Sinha, V. Barger, Midi-review: Status of weak scale supersymmetry after LHC Run 2 and ton-scale noble liquid WIMP searches, Eur. Phys. J. Special Topics 229, 3085 (2020)

4. B. Mukhopadhyaya, First an idol, then an outcast: Both for wrong reasons? Eur. Phys. J. Special Topics 229, 3143 (2020)

5. R.K. Barman, G. Belanger, R.M. Godbole, Status of low mass LSP in SUSY, Eur. Phys. J. Special Topics 229, 3159 (2020)

6. K. Huitu, A minimal supersymmetric left-right model, dark matter and signals at the LHC, Eur. Phys. J. Special Topics 229, 3187 (2020) 
7. M. Frank, Evading $Z^{\prime}$ boson mass limits in $U(1)^{\prime}$ supersymmetric models, Eur. Phys. J. Special Topics 229, 3205 (2020)

8. E.J. Chun, Axino phenomenology, Eur. Phys. J. Special Topics 229, 3221 (2020)

9. R.N. Mohapatra, No Axion Solution to Strong CP using Parity and Supersymmetry, Eur. Phys. J. Special Topics 229, 3229 (2020)

10. M.K. Parida, R. Samantaray, Unification Predictions With or Without Supersymmetry, Eur. Phys. J. Special Topics 229, 3243 (2020)

11. D.E. López-Fogliani, C. Muñoz, Searching for Supersymmetry: The $\mu \nu$ SSM, Eur. Phys. J. Special Topics 229, 3263 (2020) 